\title{
Inverse Force Determination on a Small Scale Launch Vehicle Model using a Dynamic Balance
}

\author{
Christina L. Ngo** \\ Aerospace Computing Inc., Mountain View, California, 94043, USA \\ Jessica M. Powell ${ }^{\dagger}$ \\ NASA Johnson Space Center, Houston, TX, 77058, USA \\ James C. Ross ${ }^{\ddagger}$ \\ NASA Ames Research Center, Moffett Field, CA, 94035, USA
}

\begin{abstract}
A launch vehicle can experience large unsteady aerodynamic forces in the transonic regime that, while usually only lasting for tens of seconds during launch, could be devastating if structural components and electronic hardware are not designed to account for them. These aerodynamic loads are difficult to experimentally measure and even harder to computationally estimate. The current method for estimating buffet loads is through the use of a few hundred unsteady pressure transducers and wind tunnel test. Even with a large number of point measurements, the computed integrated load is not an accurate enough representation of the total load caused by buffeting. This paper discusses an attempt at using a dynamic balance to experimentally determine buffet loads on a generic scale hammer head launch vehicle model tested at NASA Ames Research Center's 11' $x$ 11' transonic wind tunnel. To use a dynamic balance, the structural characteristics of the model needed to be identified so that the natural modal response could be and removed from the aerodynamic forces. A finite element model was created on a simplified version of the model to evaluate the natural modes of the balance flexures, assist in model design, and to compare to experimental data. Several modal tests were conducted on the model in two different configurations to check for non-linearity, and to estimate the dynamic characteristics of the model. The experimental results were used in an inverse force determination technique with a psuedo inverse frequency response function. Due to the non linearity, the model not being axisymmetric, and inconsistent data between the two shake tests from different mounting configuration, it was difficult to create a frequency response matrix that satisfied all input and output conditions for wind tunnel configuration to accurately predict unsteady aerodynamic loads.
\end{abstract}

\section{Nomenclature}

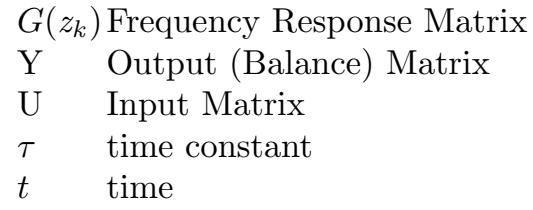

${ }^{*}$ Research Engineer, Experimental Aero-Physics Branch, Moffett Field, CA

${ }^{\dagger}$ Engineer, Applied Aeroscience \& CFD Branch.

¥Senior Aerospace Engineer, Experimental Aero-Physics Branch, Moffett Field, CA 


\section{Introduction}

In 2016, a scale model of a generic hammer head launch vehicle was tested at NASA Ames Research Center's 11'x11' wind tunnel. The main goal of the test was to evaluate the use of unsteady Pressure Sensitive Paint (uPSP) to estimate the Buffet Forcing Function (BFF), which is a time varying load used to quantify fluctuating aerodynamic loads in the transonic regime. ${ }^{2}$ Validation was performed by comparing with BFFs computed using two other techniques. The first used integrated discrete measurements from unsteady pressure transducers and the second used loads measured from an unsteady metric balance. This paper will present the results from the unsteady balance and discuss the approach taken in an attempt to remove structural modes from the balance measurements.

The generic hammer head launch vehicle configuration first tested by Coe and Nute ${ }^{1}$ in 1962 was used to investigate different techniques for measuring buffet forcing functions. Traditionally, several hundred unsteady pressure transducers are used to measure fluctuating pressures by integrating the measured pressure over a surface. Due to the limited surface area and internal volume on most wind tunnel models, the number of transducers can be well below the number required to generate accurate estimates of buffet loads without sophisticated correction schemes. ${ }^{4}$ Because of this, the estimates used for design are often overly conservative adding unnecessary weight during vehicle design.

\section{Background}

Strain gauges installed in carefully placed configurations, also referred to as balances, are used primarily to obtain steady state force and moment measurements during wind tunnel model tests. In this case, a 4-component (two force and two moment strain gauge sensors) balance was used to measure unsteady forces and moments acting on a small section of the Coe and Nute Model 11 configuration. ${ }^{1}$ During wind tunnel tests, the majority of wind tunnel models are susceptible to some vibration, either from the model itself or from the model mount. This vibration causes unwanted inertial load measurements, due to the relative movement between the model (the free, or metric end of the balance) and the sting mount (the mounted, or non-metric, end of the balance). The majority of the energy acting on the strain gauges other than the aerodynamic forces is from model and mounting structural modes.This signal contamination can be very severe, especially if the fluctuating aerodynamic load frequencies are around the model's balance natural modes. Because of this, balances tend to be unreliable when used to measure dynamic forces and moments. The present study was undertaken to look at ways to obtain an accurate measurement of the unsteady aerodynamic forces and moments that occur on a vibrating wind tunnel model, and to investigate the the pitfalls associated with noisy data or non-linear structural modes.

\section{Test Overview}

The test article, a model of a hammer head launch vehicle described previously in Coe and Nute, ${ }^{1}$ was tested in the NASA Ames Research Center's 11' Transonic Wind Tunnel.This model was chosen because it is in the open literature and the shape is similar to current payload fairing configurations. Details of the model 
configuration are shown in Figure 1. ${ }^{2}$

The hammer head configuration generatesd a broadband fluctuating load. ${ }^{1}$ A dynamic Finite Element Model (FEM) was constructed for the balance. The results indicated the presence of several natural vibrational modes within the expected vibrational frequency range. To investigate this in further detail, a ground vibration test (GVT) was performed on the model.

The first test had the model installed on a shaker table vertically and the $2^{\text {nd }}$ configuration had the model mounted horizontally, just as tested in the tunnel. The GVT was conducted at NASA Ames' Engineer Evaluation Lab (EEL) which had a large shake table for the model to mount vertically. The model was checked for asymmetry and large non-linear characteristics. Initially, the second modal test in the wind tunnel was solely to provide a check incase there were large changes in natural modes. Unfortunately, there were large variation in the frequency response function between the two configurations and only the wind tunnel modal test data was usable in determining unsteady aerodynamic forces. Finally, the results from the pseudo-inverse frequency response estimate were compared with spectral data obtained from unsteady pressure paint (uPSP) integrated load results.

\section{III.A. Wind Tunnel Test Matrix}

There were multiple types of data points taken during the test, but only the subset of the data points in Table 2 of the Appendix are relevant to this report. Wind-on test conditions in this analysis included 0.6 and 1.2; pitch angles $-4,0$, and 4; and Reynolds numbers of 2,3 , and $5 x 10^{2}$. To acquire relevant data, the model was outfitted with a variety of sensors, including uPSP, unsteady pressure transducers mounted in the model skin, the dynamic balance, and various accelerometers.

III.B. Model

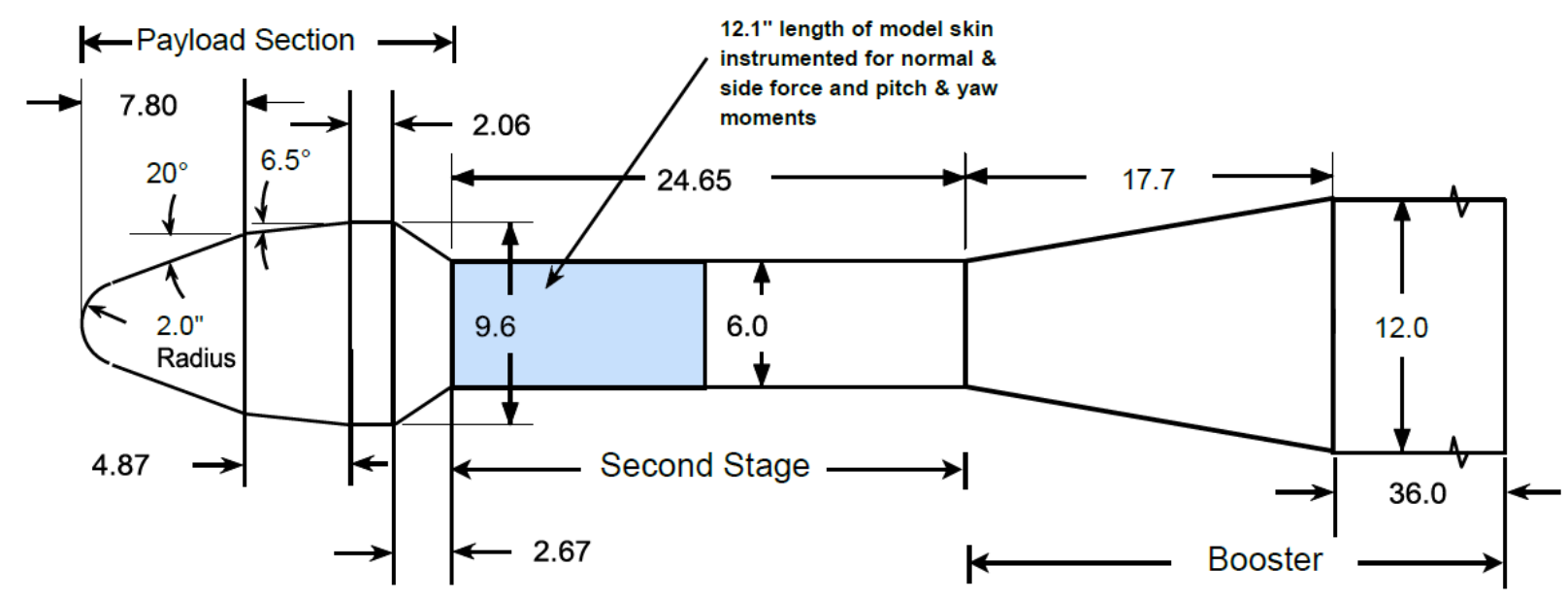

Figure 1: Model Dimensions (Inches)

Figure 1 shows wind tunnel model dimensions in inches. The portion shaded in blue indicates the metric segment of the model located on the second stage of the launch vehicle. The test article was instrumented with the following sensors. The instruments with an asterisk "*" were a part of the unsteady balance BFF analysis.

- 4 component balance* 
- 3 Tri-axis Accelerometers 4 Endevco Model 65 Isotron accelerometers*

- 1 Tri-axis Accelerometer Kistler Model 8792A100

- 211 Unsteady Pressure Transducers

- 4 Resistance Temperature Detectors (RTDs)*

- 1 Inertial Angle Sensor QA-2000 Honeywell*

- Unsteady Pressure Sensitive Paint for time-accurate full surface pressure measurements

- High-speed Shadowgraph

- Infrared Thermography for transition detection

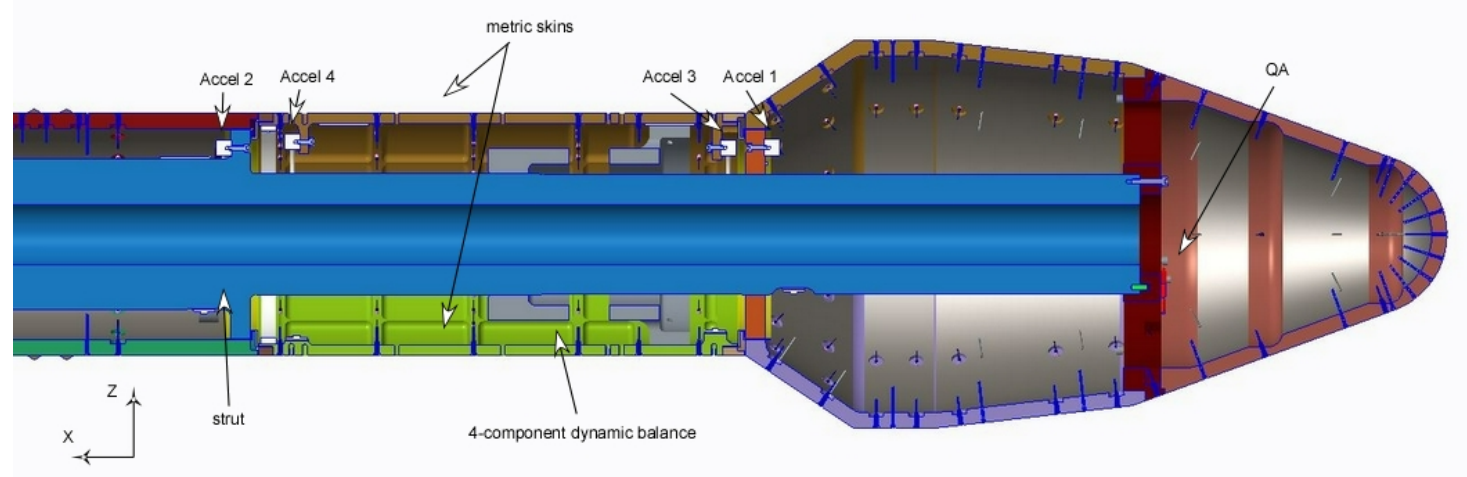

Figure 2: Model Instrumentation

Accelerometer 3 and 4 were located on the metric skin and in close proximity, accelerometer 1 and 2 was located on the non-metric end at the sting flange and orange wheel shown in Figure 2. A fifth accelerometer was attached near where the sting and strut met to monitor strut vibrations. The 4-component balance was used to measure normal and side forces, as well as pitch and yaw moments.

\section{III.C. Data Acquisition}

The accelerometers, 4 component balance, and RTDs were all sampled at $10 \mathrm{kHz}$ with a sigma delta filter. ${ }^{3}$ The Endevco Model 65 accelerometers located on or near the metric skin were the most critical for the modal test. The sensors were sent out to the manufacturer for calibration to test frequency vs. acceleration sensitivity. With the resulting calibration, a frequency dependent correction was applied to all the Endevco accelerometers. It is well known that piezo electric accelerometers are sensitive to temperature changes therefore RTDs were superglued to accelerometer 1-4 to monitor any temperature fluctuations. All dynamic data were acquired by NASA Ames Research Center's Unitary Wind Tunnel's Dynamic Data System (DDS).

\section{Modal Analysis}

To remove the inertial loads due to structural modes, the balance and model dynamic characteristics were experimentally determined. The main objective of the modal tests was to determine balance flexure response to create a set of frequency response matrices. Two sets of tests were performed on the model with design variations due to hardware limitations and mounting capabilities on the shaker table. The initial plan was 
to conduct a ground vibration test with a shaker table to fully characterize the metric volume of the model. The second GVT on the model in the wind tunnel was to check for structural changes in case instrumentation were shifted during model install. Ideally, the entire GVT effort would have been completed in the wind tunnel as tested. The shaker setup and mounting holes on the metric skin was limited to only accepting input loads at $90^{\circ}$ and $180^{\circ}$. In addition, the small portable shaker used in the wind tunnel provided less than $20 \%$ of allowable balance force input resulting in low signal to noise ratio.

\section{IV.A. Modal Analysis Configurations}

A ground vibration test was performed on the top half of the model containing the metric balance. An additional custom mount was designed allowing mounting of the model on a shake table at the Engineering Evaluation Lab (EEL), NASA Ames Research Center. The shaker table provides a single direction load on the model that is controlled by a signal generator. The model was tested with a random and sine chirp input in five directions.

The sting mount for the model was designed into two parts. The top half was mounted to a circular plate that adapted to the 3 ton shaker table shown in Figure 3. Instrumentation cables were tightly strapped down to the sting making sure there was minimal movement. The top half was fully assembled before balance calibration to avoid moving instrumentation cabling and wires later on. The skins were not removed again untill after completion of the test so as to not disturb the 96 transducer cables and reference tubes that bridge the balance metric break. The assumption was that the spring constant and damping of the balance/model assembly would not change. A post test static calibration of the balance verified that at least the spring constant did not measurably change.

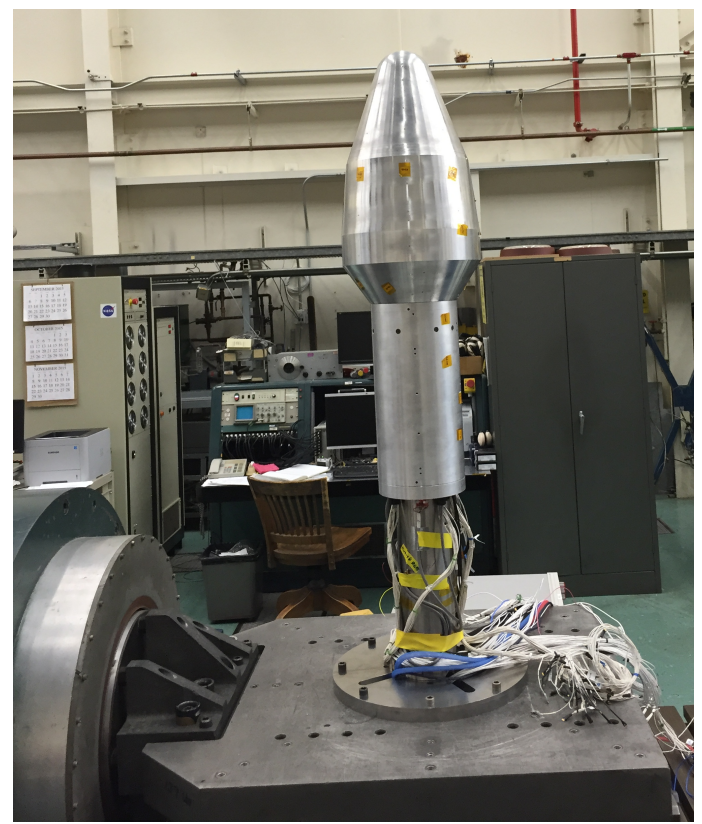

Figure 3: Engineering Evaluation Lab Modal Test Setup

A second modal test was conducted on the fully assembled launch vehicle model installed in NASA Ames 11' Wind Tunnel before and after wind-on data acquisition. The portable shaker included a BK Vibration Exciter Type 4809, custom mounting brackets, large tripod, and a PCB integrated circuit piezoelectric (ICP) force sensor. Using the mounting holes used for balance calibration, the load cell was securely fastened to the metric skin providing a rigid mount between the shaker and model metric skin with the load cell in between. 
The shaker was applied at 5 zones for z-direction oscillations and 4 zones for y-direction oscillations as shown in Figure 4a \& 5. The input load which was applied to a known distance from the balance center, was broken down into normal force and pitching moment from measured moment arm when loaded at 180 degrees. Similarly, the input load at 90 degrees was broken down into side force and yaw moment. The load cell data, 4 tri-axial accelerometers, and balance were all sampled at 10khz.

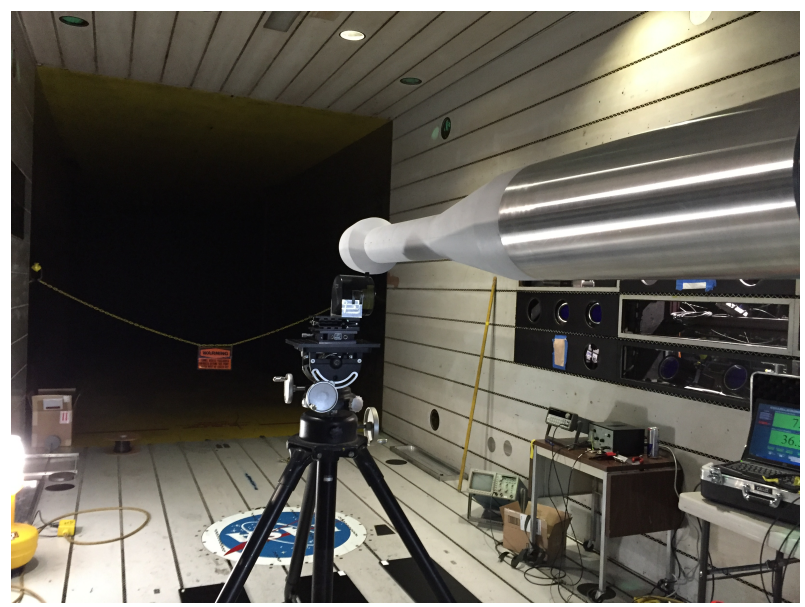

(a) Full Configuration

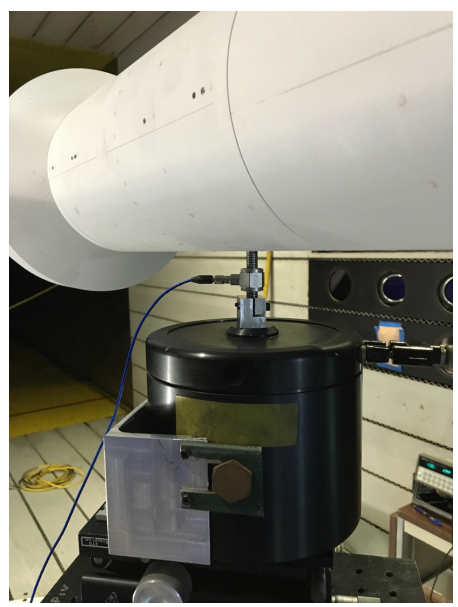

(b) Shaker Attachment

Figure 4: Wind Tunnel Modal Test Setup
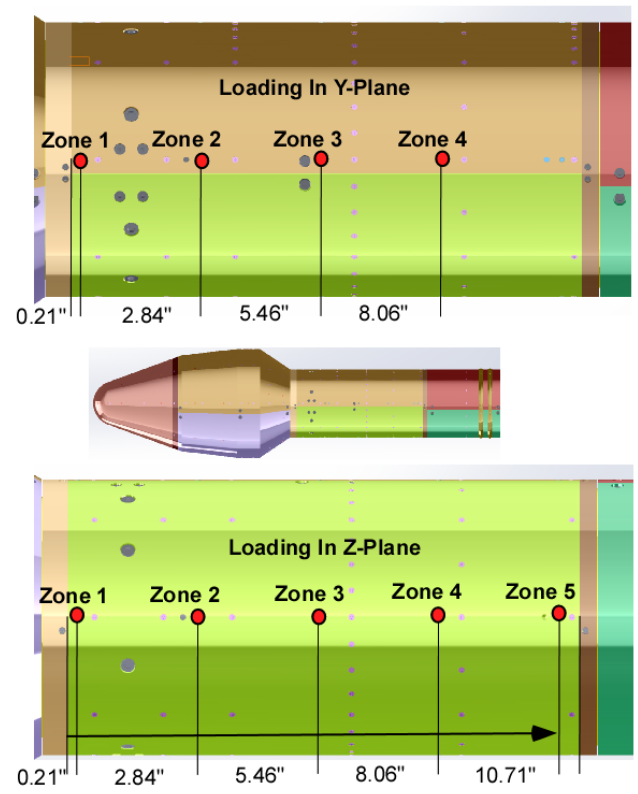

Figure 5: Loading Locations for Wind Tunnel Modal Test

\section{IV.B. Modal Analysis Results}

The modal results will be described below as well as the differences between the two tests. The resulting data from both modal test was averaged with a block size of 4096 Discrete Fourier Transform (DFT) with 
$50 \%$ overlap using Eq. (1).

$$
\mathrm{U}(k)=\frac{1}{l} \sum_{\tau=0}^{l-1} u(v) \mathrm{e}^{-j \frac{2 \pi k}{l} \tau}
$$

The input excitations were generated at different zones for each data point. The balance data were always used as the output matrix when determining the Frequency Response Function with Eq. (2) where Eq. (3) is the frequency response matrix and Eq. (6) is the error, usually caused by noise. The error was assumed to be 0 by invoking the periodicity condition for random errors. To assure $\mathrm{U}(k)$ was invertible, a rich random excitation was used as the input signal. The goal of the FRF was to fully characterize the 4 flexures that bridged the metric and non-metric portion of the model.

$$
\begin{aligned}
\mathrm{Y}(k) & =G\left(z_{k}\right) U(k)-\mathcal{E}_{u} \\
G\left(z_{k}\right) & =\sum_{\tau=0}^{\infty} Y_{\tau} \mathrm{e}^{-j \frac{2 \pi k}{l} \tau} \\
z_{k} & =\mathrm{e}^{-j \frac{2 \pi k}{l}} \\
\mathrm{Y}(k) & =\frac{1}{l} \sum_{\tau=0}^{l-1} y(\tau) \mathrm{e}^{-j \frac{2 \pi k}{l} \tau} \\
\mathrm{U}(k) & =\frac{1}{l} \sum_{\tau=0}^{l-1} u(\tau) \mathrm{e}^{-j \frac{2 \pi k}{l} \tau} \\
\mathcal{E}(k) & =\frac{1}{l} \sum_{\tau=0}^{\infty} Y_{\tau} \mathrm{e}^{-j \frac{2 \pi k}{l} \tau} \mathrm{e}_{\tau}(k)
\end{aligned}
$$

The model was rotated about its center axis at $0^{\circ}, 45^{\circ}, 90^{\circ}$, and $135^{\circ}$ to check if input directions were consistent with peak response levels. Table (1) shows the different model configuration and input excitation.

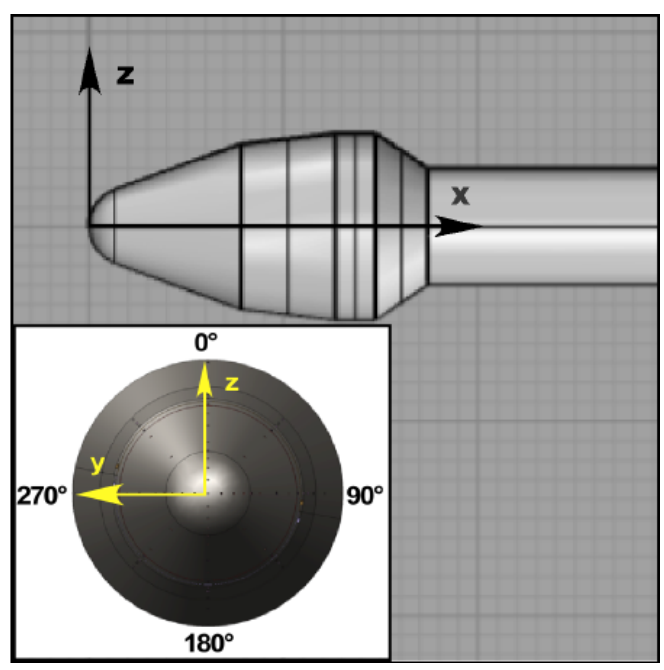

Figure 6: Model Coordinate System 
Table 1: Shaker Table Modal Test Run Matrix

(a) Sweeps

\begin{tabular}{cc}
\hline Vibration Directions & Load Levels $(\mathrm{g})$ \\
\hline$(0,45,90,135)$ & $(0.25,0.5,0.6)$
\end{tabular}

(b) Random

\begin{tabular}{cc}
\hline Vibration Directions & Load Levels (grms) \\
\hline$(0,45,90,135)$ & $(0.8,1.011)$
\end{tabular}

The first tests completed on the shaker table showed configuration repeatability for both a sweep and random input signal. This was done for all configurations, at a variety of input locations and magnitudes. For a random input GVT test configuration, Figures $7 \mathrm{a}$ and $7 \mathrm{~b}$ show repeated data sets taken at EEL, with input direction at $0^{\circ}$ and random input excitation level at 1.011 grms from 5 to $2 \mathrm{khz}$. The peak responses and frequency locations do not vary between Experiment 1 and Experiment 2.

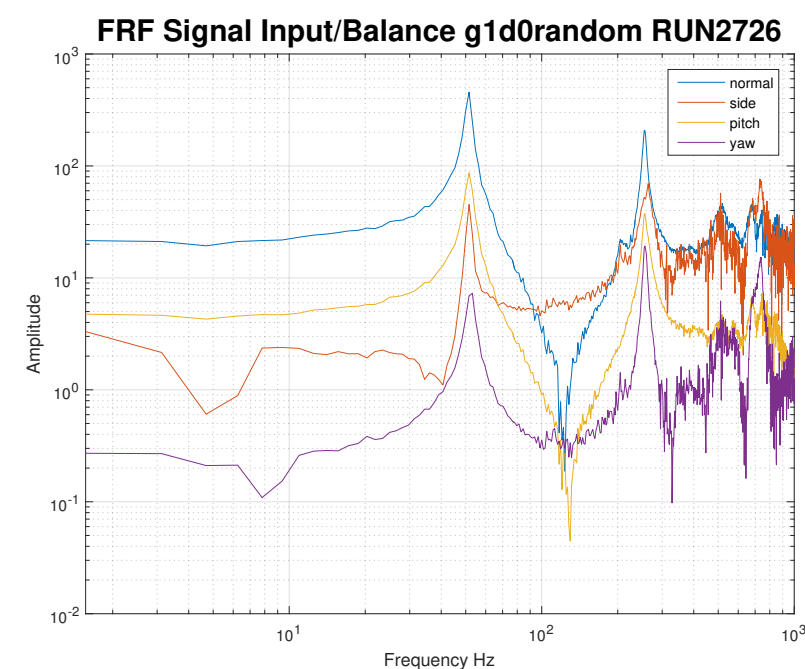

(a) Experiment (1)

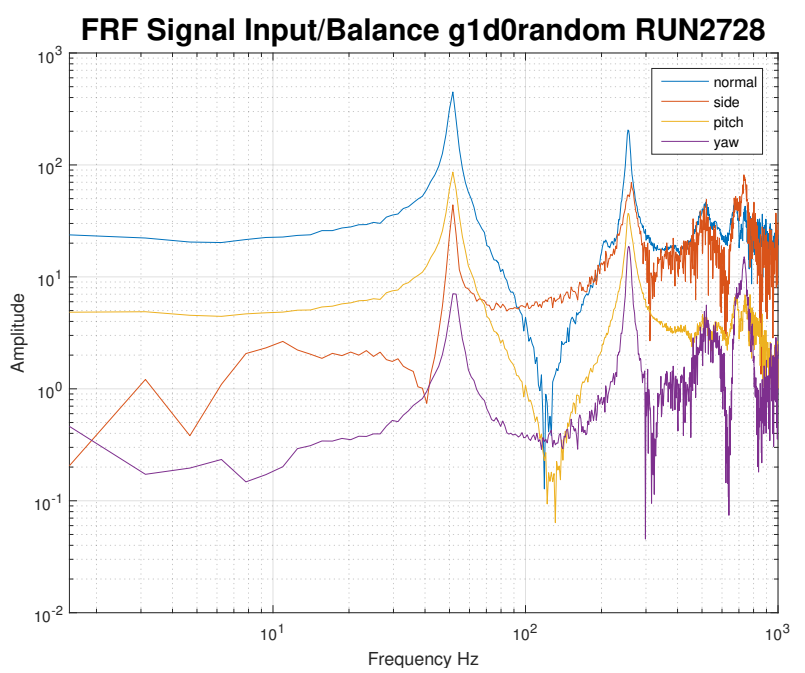

(b) Experiment (2)

Figure 7: Shaker Table Modal Test Random Input Data Repeats (Direction: $0^{\circ}$ )

The repeat points were checked for structural changes and noise variation that may cause problems during post processing. An indication of structural non-linearity is when magnitude of peaks in the frequency domain vary with excitation load. Figure $8 \mathrm{a}$ and $8 \mathrm{~b}$ show that the frequency response functions were consistent at varying levels of grms in the same loading direction which indicated the flexures were structurally linear. 
FRF Signal Input/Balance g1d0random RUN2730

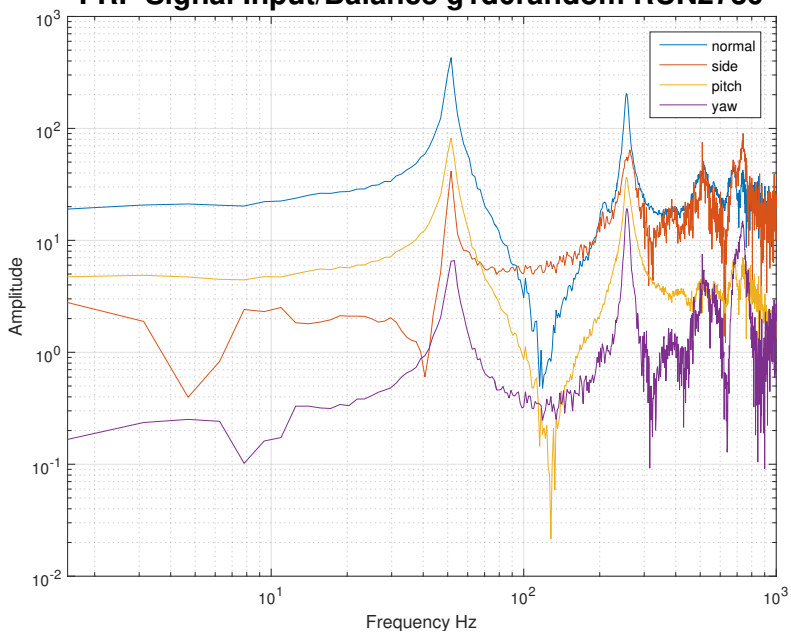

(a) Experiment (1) 1.011 grms
FRF Signal Input/Balance g80d0random RUN2724

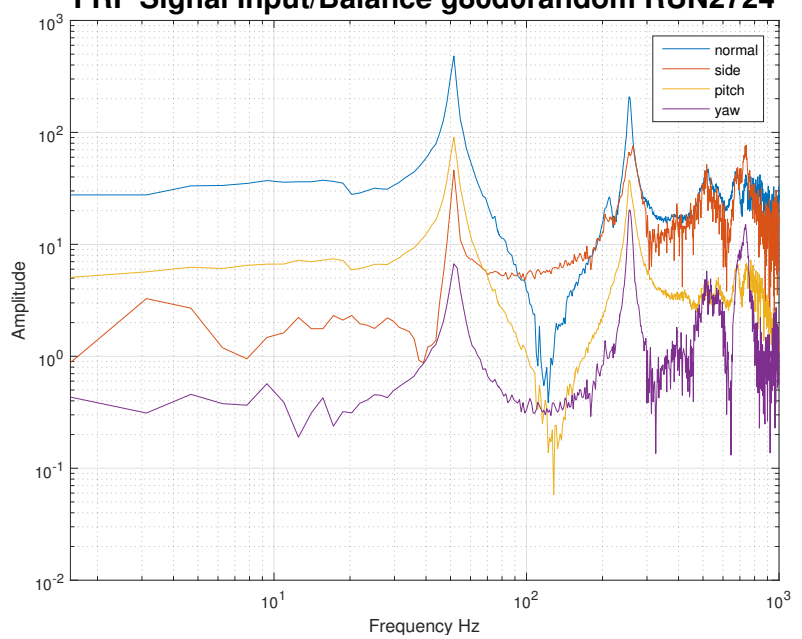

(b) Experiment (2) 0.8 grms

Figure 8: Shaker Table Modal Test; Varying grms (Direction: $0^{\circ}$ )

The shaker table modal test results were compared to the wind tunnel configuration modal test results shown in Figure 9a and 9b. There were significant differences in the modes at the lower end of the spectrum (below $300 \mathrm{~Hz}$ ). The peak at $255 \mathrm{~Hz}$ was likely due to the mounting configuration on the shaker table given the mode only showed up on shaker table configuration and not in the wind tunnel. The lower frequency peak at $50 \mathrm{~Hz}$ was also likely due to mounting and orientation of the model. NASA Ames Unitary Tunnel internal studies has shown that the 11' model mounting strut has known structural modes around $11 \mathrm{~Hz}$ and $22 \mathrm{~Hz}$ which can be seen in a subset of GVT wind tunnel modal test data. For example, Figure 9a and 9b peaks at $12.2 \mathrm{~Hz}$ and $25.6 \mathrm{~Hz}$ were due to the strut modes propagating into the model. The $60 \mathrm{~Hz}$ peaks were caused by electromagnetic interference (EMI) disturbance from external sources. The modes around $670 \mathrm{~Hz}$ and $758 \mathrm{~Hz}$ were flexure modes from the balance which stayed consistent between the two modal tests on the shaker table and in the wind tunnel test section even with negligle variation due to model orientation. 


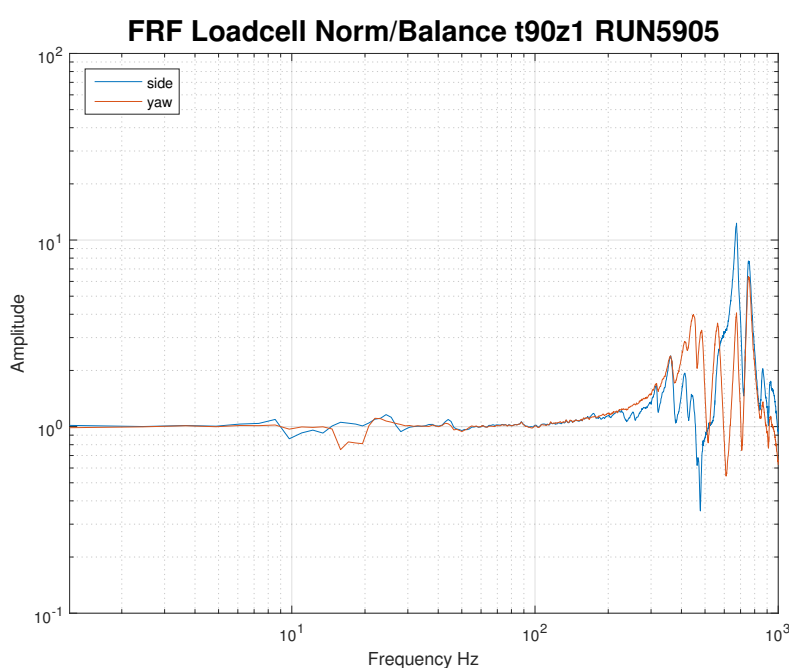

(a) Experiment (1) Input Excitation at $90^{\circ}$

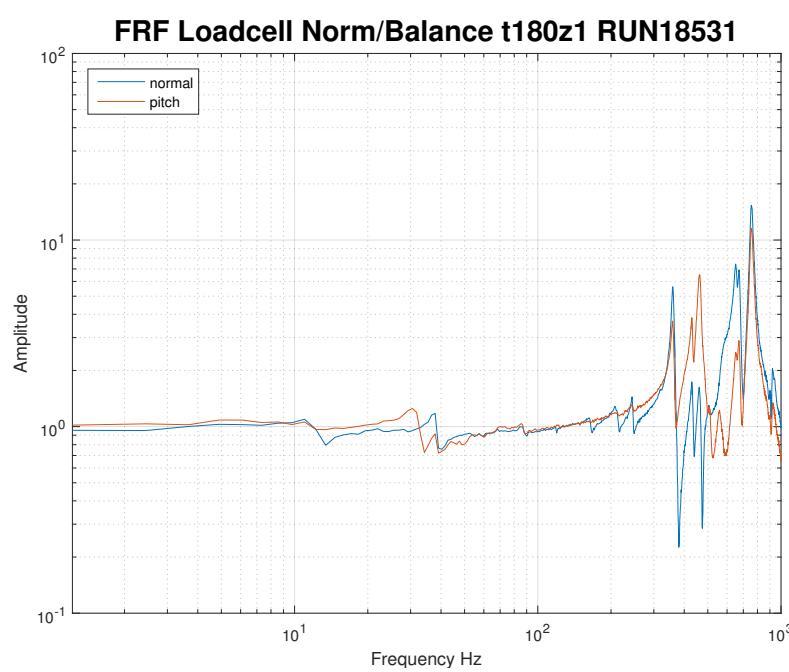

(b) Experiment (2) Input Excitation at $180^{\circ}$

Figure 9: Wind Tunnel Modal Test; Random Input Data at Zone 1 (Direction: $90^{\circ}$ and $180^{\circ}$ )

Figure 10a 10b, 11a, and 11b show balance output magnitude variation with excitation direction. The peak response for normal force and pitching moment was anticipated to be at $0^{\circ}$ because they were applied directly in the plane of normal force strain gauge deflection. Similarly, the lowest peak response for normal force and pitching moment were anticipated to be at $90^{\circ}$. Instead, the normal force showed abnormal responses with peak response at $45^{\circ}, 70 \%$ of peak at $0^{\circ}$ and $90^{\circ}$, and low peak response at $135^{\circ}$. The side force and yawing moment performed as expected with peak responses at $90^{\circ}$ input excitation and $70 \%$ of peak at $45^{\circ}$.

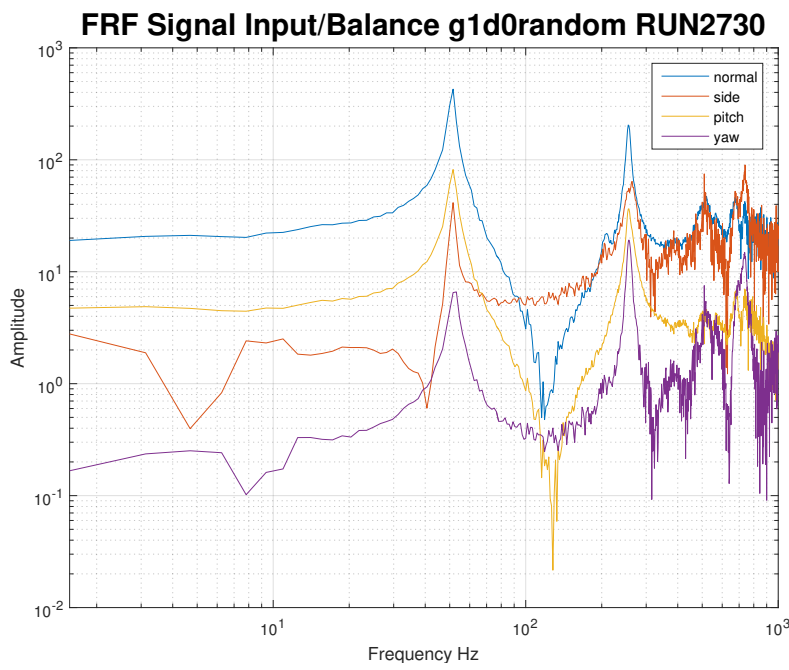

(a) Experiment (1) Input Excitation at $0^{\circ}$

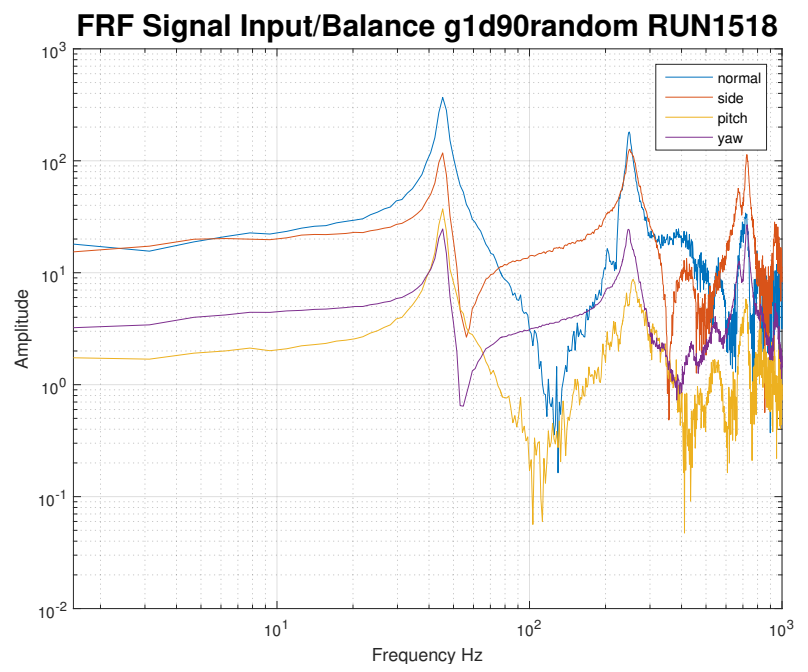

(b) Experiment (2) Input Excitation at $90^{\circ}$

Figure 10: Shaker Table Modal Test Random Input Data Magnitude Comparison (Direction: $0^{\circ}$ and $90^{\circ}$ ) 


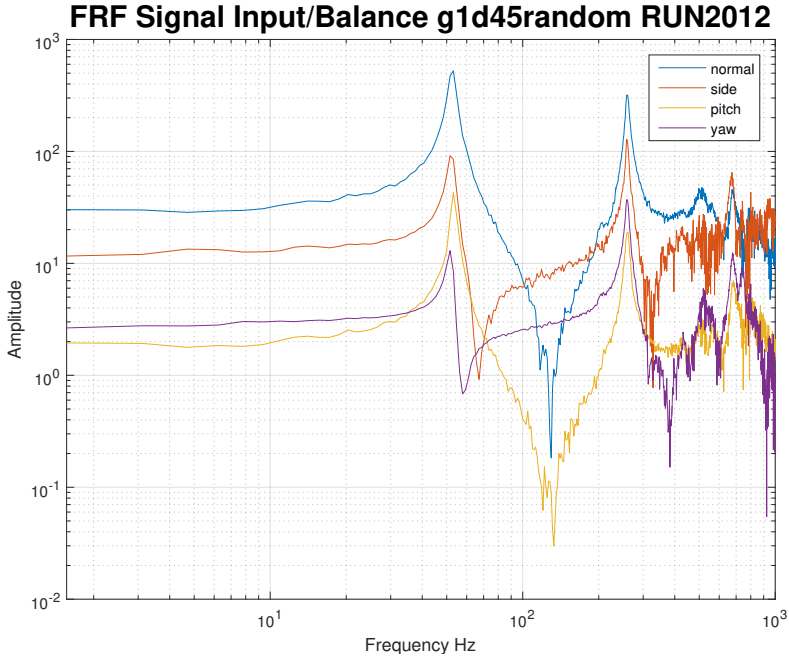

(a) Experiment 3 Input Excitation at $45^{\circ}$

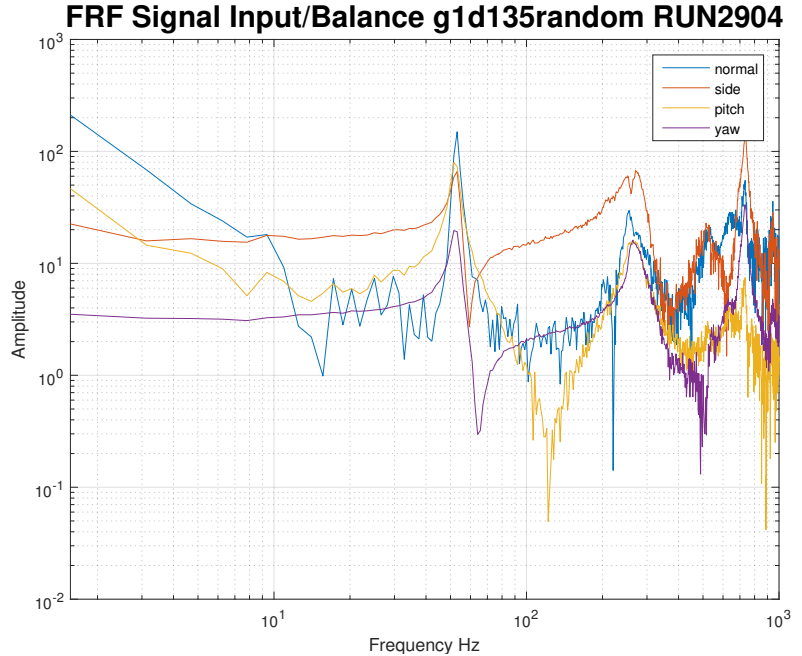

(b) Experiment 4 Input Excitation $135^{\circ}$

Figure 11: Shaker Table Modal Test Random Input Data Magnitude Comparison (Direction: $45^{\circ}$ and $135^{\circ}$ )

The flexure response from the experimental data was then compared with results computed from the CREO FEM dynamic analysis. Figure 12 shows that flexure response peak locations roughly approximated with the experimental data. Specifically, the computational results estimated the lower mode to be shifted $35 \mathrm{~Hz}$ lower and the second mode to be shifted $150 \mathrm{~Hz}$ higher. This was presumed to be caused by the simplified FEM model, which did not incorporate the additional weight, stiffness, and damping from fasteners and instrumentation such as kulites, accelerometers, cabling, and tubing.

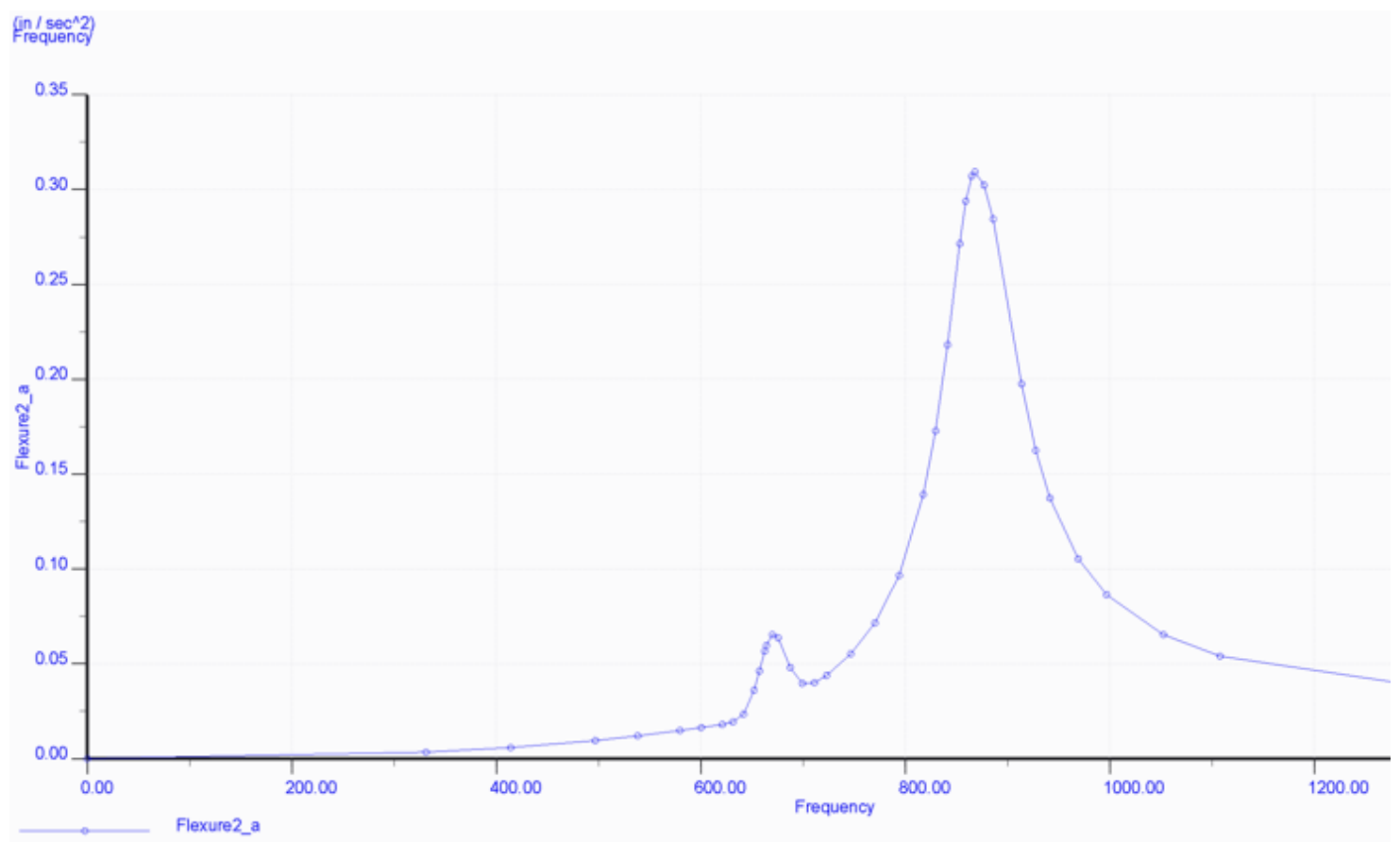

Figure 12: FEM Modal Analysis 


\section{Results}

An indirect force estimation was applied by using a pseudo-inverse of the frequency response matrix to predict input loads or in this case, aerodynamic loads.

$$
\begin{aligned}
\mathrm{X}(k) & =G(k)^{\dagger} \mathrm{Y}(k) \\
G(k)^{\dagger} & =\left[G(k)^{H} G(k)\right]^{-1} G(k)^{H}
\end{aligned}
$$

Equation (7) was used to estimate the integrated forces around the metric section with a single input excitation and 4 strain gauges response located on the balance flexures. A known limitation of this technique, is associated with the possibility that the problem is ill-conditioned because the number of degrees-of-freedom in the excitation frequency range are less than the number of estimated forces. In general, it is difficult to model a set of Frequency Response Matrix that satisfies all the conditions of modal tests.

Figure 13a and 13b shows the corrected balance output results using Equation (7) to estimate the BFF at Mach 0.85, alpha 4 degrees, and Reynolds 3 million/ft. The raw data (blue) represents the uncorrected balance output, the corrected data (red) represents the BFF estimate from the pseudo inverse FRF force determination method, and the UPSP data (yellow) represents the integrated loads from a separate measurement technique. The integrated UPSP results, because they were completely independent of the balance, were assumed to be the validation reference data set. If the psuedo-inverse technique was perfectly correct, the yellow and red traces should overlay at all frequencies.
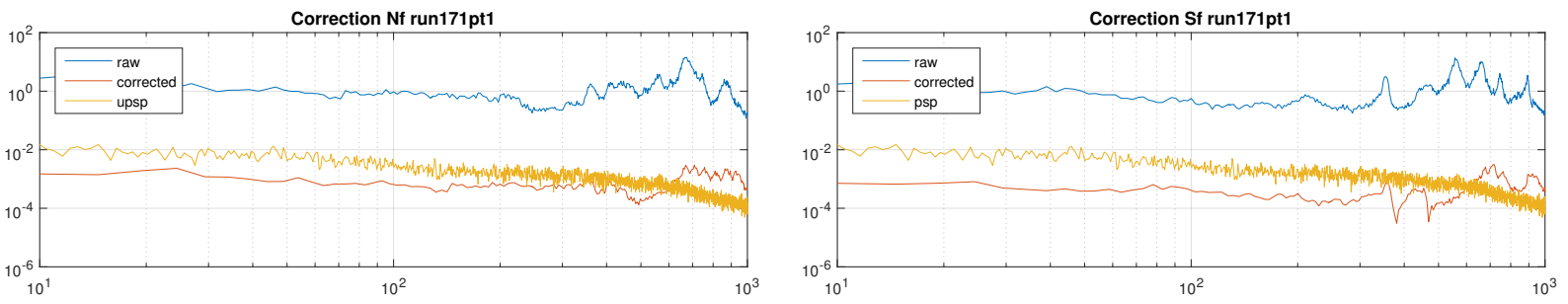

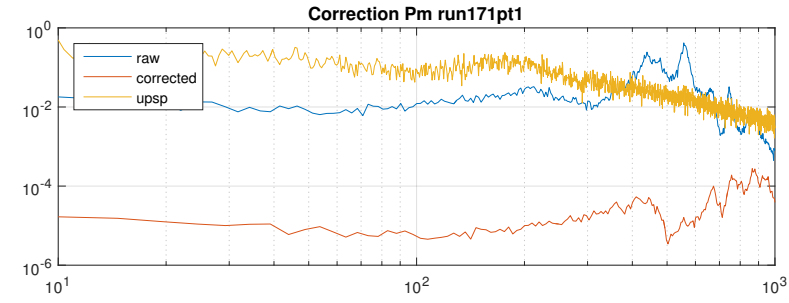

(a) Normal Force and Pitching Moment

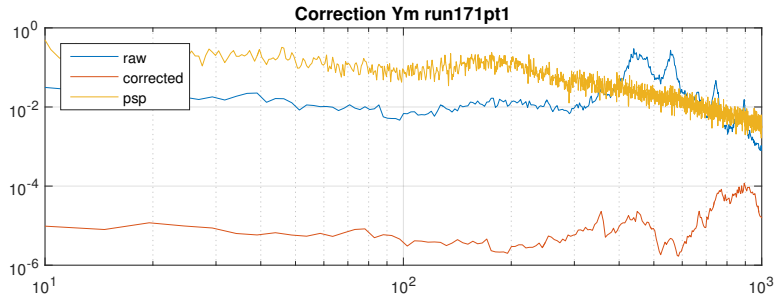

(b) Side Force and Yawing Moment

Figure 13: Run 171 Sequence 01 Balance Corrections

Of interest in Figure 13a and 13b, in the direct normal and side force load corrections, the corrected results roughly approximated the UPSP amplitudes. Note however, the pitch and yaw moment corrected amplitudes deviate substantially from the UPSP estimates. Thus the BFF estimates approximate the direct force better than they do the moment. In fact, the UPSP moment data is more closely approximated by the raw data compared to the corrected data. This suggest that the raw data possessed minimal energy content associated with structural dynamic modes.Depending on the location of the input loads, a different mode shape can be excited. Thus, the deviation seen in the corrected moment data and UPSP could potentially be caused by exciting various bending modes. 


\section{Conclusion}

The modal analysis showed the model modes varied between the Engineering Evaluation Lab and Wind Tunnel configuration. The flexure responses at $670 \mathrm{~Hz}$ and $758 \mathrm{~Hz}$ were consistent between the two tests for the same load input direction. The results from the wind tunnel and shaker table modal tests showed the model was relatively linear and axisymmetric at all increments of $90^{\circ}$, where all four balance flexures were at the same orientations. Similarly, the balance flexures were at the same orientation in increments of $45^{\circ}$. Unlike the increments of $90^{\circ}$ modal test cases, there were frequency peak differences between the $45^{\circ}$ and $135^{\circ}$ cases for normal force and pitching moment indicating large non-linear characteristics.

Due to the non linearity, the model not being axisymmetric, and inconsistent data between the two shake tests due to mounting configuration, it was difficult to create a frequency response matrix that satisfied all input and output conditions for wind tunnel configuration to accurately predict unsteady aerodynamic loads.

During the wind tunnel data corrections, the normal and side force load corrections results roughly approximated the UPSP amplitudes. In contrast, the pitch and yaw moment corrected amplitudes deviated substantially from the UPSP estimates. The raw data was more closely approximated by the UPSP moment data, this suggests that the raw data possessed minimal energy content associated with structural dynamic modes. Thus, the deviation seen in the corrected moment data and UPSP could potentially be caused by unintentionally exciting various bending modes.

\section{Future Work}

The balance matrix used to compute forces and moments from strain gauge output was treated as a black box for this paper and incorporated in the frequency response function matrices. Instead of using the computed forces, the psuedo inverse frequency reponse function force determination method will be applied directly to strain gauge outputs. This will simplify the investigation of the non-axisymmetric model and flexure dynamic characteristics. In addition, a force determination technique using an inverse Markov parameter and forward observer gain method is currently being investigated to more accurately estimate unsteady aerodynamic loads. 


\section{Appendix}

Table 2: DDS+uPSP Test Matrix

\begin{tabular}{|c|c|c|c|c|c|c|}
\hline Run & \# of Points & Mach & Rey (mil) & Alpha & Beta & Config \\
\hline 84 & 3 & 0.80 & 2 & $\mathrm{~A} 1$ & 0.0 & baseline \\
\hline 85 & 3 & 0.92 & 2 & $\mathrm{~A} 1$ & 0.0 & baseline \\
\hline 86 & 3 & 1.02 & 2 & $\mathrm{~A} 1$ & 0.0 & baseline \\
\hline 87 & 3 & 1.02 & 2 & $\mathrm{~A} 1$ & 0.0 & baseline \\
\hline 100 & 3 & 0.60 & 3 & A1 & 0.0 & baseline \\
\hline 101 & 3 & 0.80 & 3 & $\mathrm{~A} 1$ & 0.0 & baseline \\
\hline 102 & 3 & 0.85 & 3 & $\mathrm{~A} 1$ & 0.0 & baseline \\
\hline 103 & 3 & 0.85 & 3 & A1 & 0.0 & baseline \\
\hline 104 & 3 & 0.92 & 3 & $\mathrm{~A} 1$ & 0.0 & baseline \\
\hline 105 & 3 & 1.02 & 3 & A1 & 0.0 & baseline \\
\hline 106 & 3 & 1.10 & 3 & $\mathrm{~A} 1$ & 0.0 & baseline \\
\hline 107 & 3 & 1.10 & 3 & A1 & 0.0 & baseline \\
\hline 108 & 3 & 1.10 & 3 & $\mathrm{~A} 1$ & 0.0 & baseline \\
\hline 137 & 3 & 0.60 & 5 & $\mathrm{~A} 1$ & 0.0 & baseline \\
\hline 138 & 3 & 0.80 & 5 & A1 & 0.0 & baseline \\
\hline 139 & 3 & 0.85 & 5 & A1 & 0.0 & baseline \\
\hline 140 & 3 & 0.92 & 5 & A1 & 0.0 & baseline \\
\hline 141 & 3 & 1.02 & 5 & A1 & 0.0 & baseline \\
\hline 142 & 3 & 1.02 & 5 & A1 & 0.0 & baseline \\
\hline 143 & 3 & 1.02 & 5 & $\mathrm{~A} 1$ & 0.0 & baseline \\
\hline 149 & 3 & 0.60 & 3 & A1 & 0.0 & one flange \\
\hline 150 & 3 & 0.80 & 3 & $\mathrm{~A} 1$ & 0.0 & one flange \\
\hline 151 & 3 & 0.85 & 3 & A1 & 0.0 & one flange \\
\hline 152 & 3 & 0.85 & 3 & $\mathrm{~A} 1$ & 0.0 & one flange \\
\hline 153 & 3 & 0.92 & 3 & $\mathrm{~A} 1$ & 0.0 & one flange \\
\hline 154 & 3 & 1.02 & 3 & $\mathrm{~A} 1$ & 0.0 & one flange \\
\hline 155 & 3 & 1.10 & 3 & $\mathrm{~A} 1$ & 0.0 & one flange \\
\hline 156 & 3 & 1.10 & 3 & $\mathrm{~A} 1$ & 0.0 & one flange \\
\hline 157 & 3 & 1.10 & 3 & $\mathrm{~A} 1$ & 0.0 & one flange \\
\hline 168 & 3 & 0.60 & 3 & $\mathrm{~A} 1$ & 0.0 & two flanges \\
\hline 169 & 3 & 0.80 & 3 & $\mathrm{~A} 1$ & 0.0 & two flanges \\
\hline 170 & 3 & 0.85 & 3 & $\mathrm{~A} 1$ & 0.0 & two flanges \\
\hline 171 & 3 & 0.92 & 3 & $\mathrm{~A} 1$ & 0.0 & two flanges \\
\hline 172 & 3 & 1.02 & 3 & A1 & 0.0 & two flanges \\
\hline 173 & 3 & 1.10 & 3 & $\mathrm{~A} 1$ & 0.0 & two flanges \\
\hline
\end{tabular}


Table 2 - DDS+uPSP Test Matrix, Continued

\begin{tabular}{|ccccccc|}
\hline Run & \# of Points & Mach & Rey (mil) & Alpha & Beta & Config \\
\hline 174 & 3 & 1.10 & 3 & A1 & 0.0 & two flanges \\
175 & 3 & 1.10 & 3 & A1 & 0.0 & two flanges \\
176 & 3 & 0.85 & 3 & A1 & 0.0 & two flanges \\
\hline
\end{tabular}

The sweeps are:

- M1: Mach $=0.7,0.8,0.85,0.88,0.9,0.92,0.95,0.98,1.02,1.05,1.1,1.15,1.2$

- $\mathrm{M} 2:$ Mach $=0.8,0.6$

- A1: Alpha = -4.0, 0.0, 4.0

- AB1:

\begin{tabular}{|c|c|c|c|c|c|c|c|c|c|c|c|c|c|}
\hline Alpha & 0.0 & -2.8 & 2.8 & 0.0 & -0.7 & 0.7 & -4.0 & -1.0 & 0.0 & 1.0 & 2.0 & 3.0 & 4.0 \\
\hline Beta & -4.0 & -2.8 & -2.8 & -1.0 & -0.7 & -0.7 & 0.1 & 0.1 & 0.1 & 0.1 & 0.1 & 0.1 & 0.1 \\
\hline
\end{tabular}

\section{Acknowledgements}

The test team would like to extend their thanks to the NASA Engineering Support Center (NESC), who provided financial and technical support for this project. The authors would also like to thank the staff at NASA Ames Research Center's Engineering Evaluation Lab (EEL) and Unitary Wind Tunnel Facility, who helped with collecting and interpreting modal data.

\section{References}

${ }^{1}$ Coe, C. F. and Nute, J. B. Steady and Fluctuating Pressures at Transonic Speeds on Hammerhead Launch Vehicles. NASA TM X-778, 1962.

${ }^{2}$ Schuster, D. M., Panda, J. M., Ross, J. C., Sellers, M., Roozeboom, N. H., Burnside, N. J., Sekula, M. K., Piatak, D. J., Ngo, C. L., Powell, J. M., and Kumagai, H., Investigations of uPSP and a Dynamic Loads Balance to Predict Launch Vehicle Buffet Environments, NESC RP-14-00962, July 2016.

${ }^{3}$ Park, Sangil, "Principles of Sigma-Delta Modulation for Analog to Digital Converters", Motorla APR8/D Rev 1

${ }^{4}$ Piatak, D. J., Sekula, M. K., Rausch, R. D., Ross, J. C., and Sellers, M. E.., Assessment of the Use of Unsteady Pressure Sensitive Paint for the Development of Launch Vehicle Buffet Forcing Functions, AIAA Paper 2017-xxxx, AIAA Science and Technology Forum and Exposition (SciTech 2017), Grapevine, TX, January 2017. 